\title{
MOSES - A modelling tool for the analysis of scenarios of the European electricity supply system
}

\author{
S. Weitemeyer ${ }^{1, \mathrm{a}}$, T. Feck ${ }^{1}$, and C. Agert ${ }^{1}$ \\ ${ }^{1}$ NEXT ENERGY - EWE-Forschungszentrum für Energietechnologie e.V., Oldenburg, Germany
}

\begin{abstract}
Recent studies have shown that a transition of the current power supply system in Europe to a system almost entirely based on fluctuating Renewable Energy Sources (RES) by mid-century is possible. However, most of these scenarios require a significant amount of back-up power capacities to ensure the security of electricity supply. This would imply high additional investments and operating costs. Hence, alternative options should be investigated first. Here we present a first outlook of our simulation model MOSES which will be able to analyse different target states of the European electricity system in 2050. In this model long-term meteorological data series are used to optimise the capacity mix of RES in Europe. One of the main elements of our tool is a simplified European grid model which will be used to calculate the load flows in a transnational electricity network. In addition, alternative options for reduction of additional back-up power like the expansion of the transmission grid, the use of demand-side management and/or the installation of over-capacities will be implemented. The results will be used to provide scientifically proven recommendations to policy makers for a reliable energy supply system in Europe based on Renewable Energy Sources.
\end{abstract}

\section{Introduction}

With growing concern about climate change, it is a common goal in the European Union to reduce man-made greenhouse gas emissions [1]. The two main measures that likely lead to the achievement of this goal are the improvement of energy efficiencies and the transformation of the current European power supply system to a system based on RES [2]. However, this transformation is only desirable, if the final supply system provides at least the same level of energy security and stability as the current system.

\section{Motivation \& goal}

Recent studies have shown that a transition of the current power supply system in Europe to a system almost entirely based on fluctuating RES by mid-century is possible [2-4]. However, most of these scenarios require a significant amount of storage and/or quickly adjustable gas power plants as backup power capacities to ensure the security of electricity supply. Since additional back-up power

\footnotetext{
a e-mail: stefan.weitemeyer@next-energy.de
} 
capacities based on e.g. hydrogen, synthetic methane or battery technology would imply high additional investments and operating costs, alternative options should be investigated first. This raises the question if options like the expansion of the European transmission grid, the use of demand-side management methods and/or the installation of over-capacities would contribute to a substantial reduction of back-up power. In addition, most of the recent studies neglect the interactions and synergies between the different system components.

A basis for evaluating the impact of these different options is formed by model simulations. Recent studies which have been conducted using tools currently available for the European power system indicate that a power supply which is almost entirely based on RES will be likely achievable in Europe until 2050 [3-4]. Nevertheless, these studies are not able to answer some important questions regarding our future power supply. For example, it is still uncertain how the amount of required capacities for back-up plants as well as cross-border interconnections changes if long-term meteorological data sets in the order of one decade or more are used as input data

To answer this and other research relevant questions, a new model is currently being developed in the framework of a $\mathrm{PhD}$ thesis at the German energy research centre NEXT ENERGY. The simulation model MOSES (cf. figure 1) will be able to analyse different target states of the European electricity system in 2050. By running simulations for a selected set of target scenarios, it will be possible to evaluate national build-up targets in a European context and to analyse the importance of an expansion of the European transmission grid as well as the role of energy storage devices and back-up power plants.

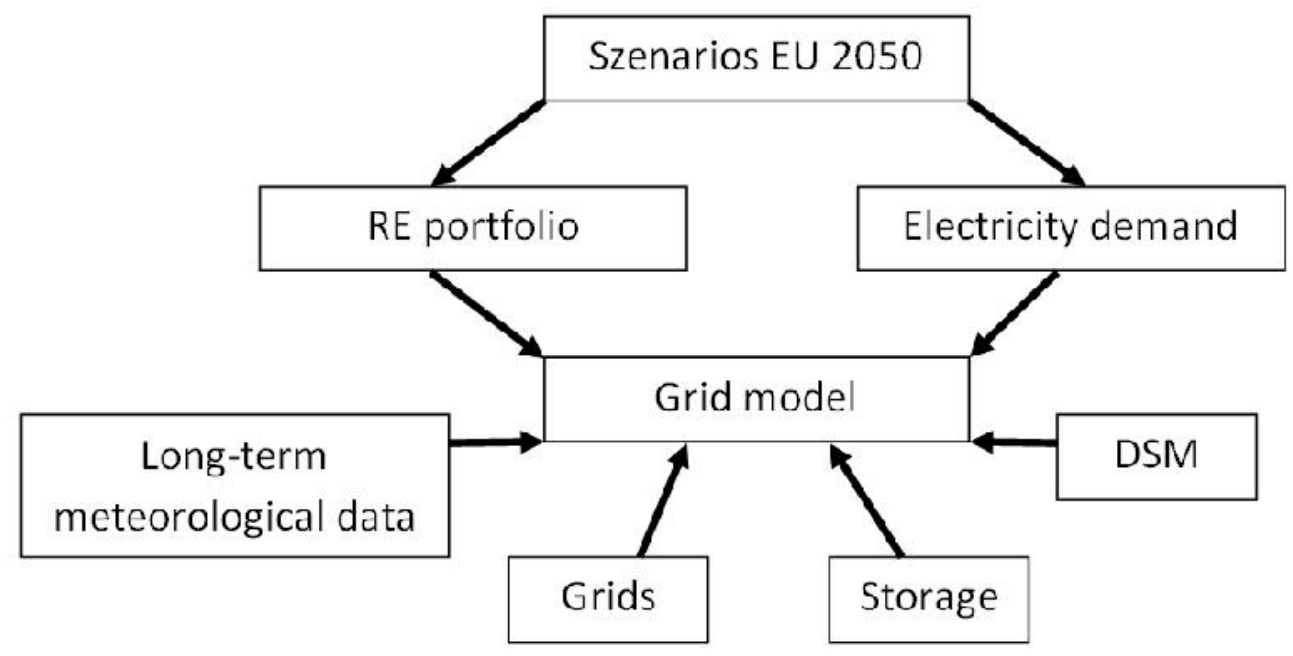

Fig. 1. Structure plan of MOSES (MOdelling tool for the analysis of Scenarios of the European electricity Supply system)

\section{Methodology}

The starting point of our model development is based on the publications of Heide et al. [5] and Hoffmann et al. [6]. In these studies, long-term meteorological data series are used to find the optimal mix of solar and wind energy generation in Europe to best match the European load curve. The results show that the amount of required storage capacities can be significantly reduced by optimizing the mix between solar and wind generation capacities in Europe. Hence, the upcoming expansion of renewable energies in Europe should be primarily based on meteorological data.

In cooperation with the university of Oldenburg, long-term meteorological data series with a high temporal $\left(\leq 1\right.$ hour for ten years) and spatial $\left(10 \times 10 \mathrm{~km}^{2}\right)$ resolution will be generated and integrated into the model. Since it is not possible to predict the exact position of every consumer and producer 


\section{$2^{\text {nd }}$ European Energy Conference}

of electricity in the target year 2050, the data will be combined in the first approach into zones with a size of the order of current TSO control areas.

A major part of the work is the development of a grid model which will be used to calculate the load flows within the European electricity grid in a simplified but still realistic way for different 2050 scenarios. Hence, it will be possible to use the model to investigate the implications of grid extensions on the overall system as well as to calculate the demand for additional cross-border interconnections.

\section{First results}

An important aspect in the development of MOSES is the implementation of a module that calculates the optimal mix of solar and wind generation in European regions. Here we show an example of a fictitious 100\% wind-plus-solar-only scenario for Eastern Germany (TSO: 50hertz) for the year 2011. For this, actual wind and solar power generation data as well as load data for the control area of $50 \mathrm{Hertz}$, all with a temporal resolution of $15 \mathrm{~min}$, were used. Since especially the installed PV capacity increased significantly over the year, both power series were scaled accordingly. Afterwards, the mismatch energy $\Delta$ was calculated according to Heide et al. using equation $1[5] . W(t)$ represents the wind power generation at time $t,\langle W\rangle$ its average over the whole year, $S(t)$ and $L(t)$ are the corresponding solar power and load time series. The parameter $a$ represents the fraction of wind in this scenario, hence the fraction for solar power is equal to $(1-a)$ in this $100 \%$ wind-plus-solar-only scenario.

$$
\Delta(t)=a \frac{W(t)}{<W>}+(1-a) \frac{S(t)}{<S>}-\frac{L(t)}{<L>}
$$

The calculation were carried out for different time steps (interval length: $15 \mathrm{~min}$, 1 day, 1 week), whereupon the averaging was done block-wise. The standard deviation $\sigma_{\Delta}$ as a measure for the balancing demand is shown in figure 2 as a function of the wind fraction $a$ for different time steps. The minimum of $\sigma_{\Delta}$ indicates the optimal mix. For the calculation with the shortest time steps ( $15 \mathrm{~min})$, the standard deviation has its minimum if the wind share fraction $a$ is $71 \%$. The standard deviation in this case is 0.75 , thus the system still requires balancing and consequently a significant amount of stored energy.

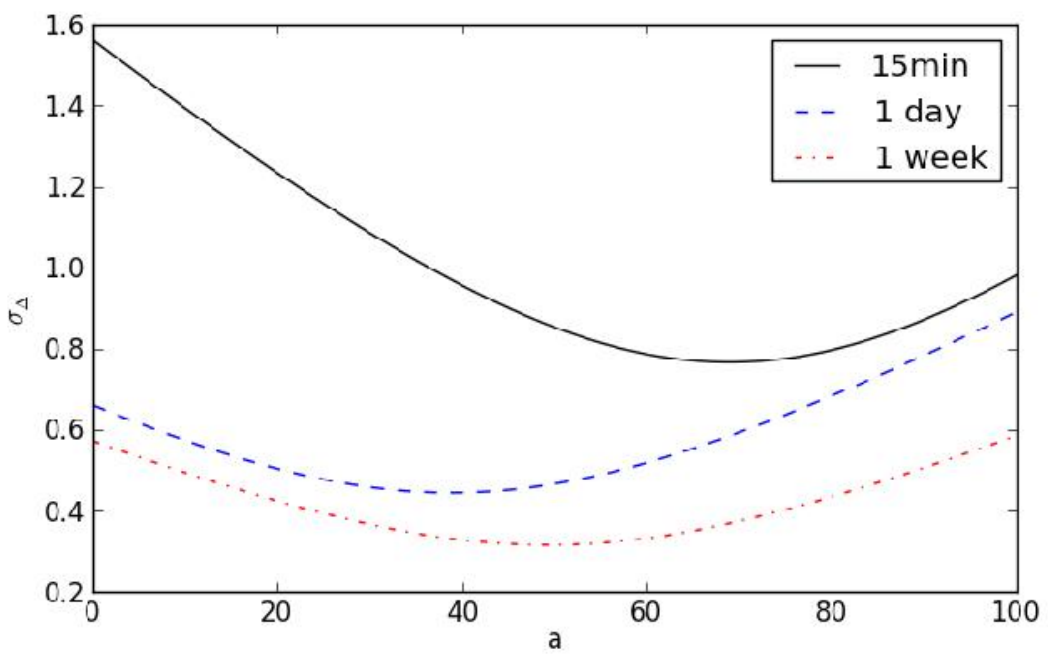

Fig. 2. Standard deviation $\sigma_{\Delta}$ of the mismatch energy as a function of the wind fraction $a$ (in percent) for different time steps in a 100\% wind-plus-solar-only scenario 
Increasing the interval length to one day significantly reduces the standard deviation. In this case the fraction of solar energy is dominating the mix. This could be explained with the difference in solar energy production between night and day vanishing on this time scale, hence the production curve gets smoother. For an interval length of one week, the standard deviation gets even smaller as in this case all short-term fluctuations are smoothed out. The results show that the choice of the time step size has a significant influence on the results and therefore has to be chosen adequately.

\section{Conclusions}

Studies have already shown that the electricity demand in a future electrify supply system can be fulfilled with RES. However, due to the high fluctuations of wind and solar power it is very likely that balancing options are essential. Using modelling tools for the simulation of a future system is an appropriate way to investigate the overall system, especially if interactions between different system components are to be taken into account. However, special attention has to be given already in the conception phase of the model to the selection of components that very likely have an impact on a future energy system (e.g. demand-side management). Due to the complexity of the system, it will be necessary to make use of simplifications, which have to be chosen appropriately. As an example, we have shown in this work that the selection of the time resolution is very likely to have a significant impact on the results. Hence, the choice of an appropriate time step will be in the scope of our upcoming work to be able to draw for instance the right conclusions regarding the need for balancing power and storage capacities in an electricity system based on Renewable Energy Sources.

\section{References}

1. European Community, Decision 2002/358/EC (2002)

2. European Climate Foundation, Roadmap 2050 - A practical guide to a prosperous, low carbon Europe (2010)

3. M. Faulstich et al., 100\% erneuerbare Stromversorgung bis 2050: klimaverträglich, sicher, bezahlbar (2010)

4. T. Klaus et al., Energieziel 2050: 100\% Strom aus erneuerbaren Quellen (2010)

5. D. Heide et al., Renewable Energy, 35, 2483-2489 (2010)

6. C. Hoffmann et al., Design of transport- and storage capacities in energy supply systems with high shares of renewable energies.(2010) 